\title{
Fixação de enfermeiras e médicos na Estratégia Saúde da Família, município de Praia Grande, São Paulo, Brasil
}

\author{
Fixedness of nurses and doctors in the Family Health Program, \\ Praia Grande, São Paulo, Brazil \\ Fijación de enfermeras y medicos de Salud de la Familia, \\ ciudad de Praia Grande, São Paulo, Brasil \\ Eliana Zulianni Lopes ${ }^{1 *}$, Aylene Emilia Moraes Bousquat ${ }^{2}$
}

\begin{abstract}
Palavras-chave: Atenção Primária à Saúde Programa Saúde da Família
\end{abstract} Recursos Humanos Primary Health Care Family Health Program Human Resources

Palabras clave: Atención Primaria de Salud Programa de Salud Familiar Recursos Humanos

\section{Resumo}

A captação, formação e fixação de profissionais são condições necessárias para a consolidação da Estratégia Saúde da Família (ESF) como modalidade estruturante da Atenção Primária à Saúde no País. 0 presente artigo teve como objetivo traçar o perfil de médicos e enfermeiras da ESF, identificando os profissionais que se fixaram à ESF no município de Praia Grande, São Paulo. Foram realizadas entrevistas semiestruturadas com todos os profissionais de nível superior em 2004-2005, e em 2007 foi verificada a permanência na ESF desses profissionais. Todas as enfermeiras e $66 \%$ dos médicos anteriormente entrevistados permaneceram atuando após 2-3 anos. Não foi observada associação estatística entre tempo de formado, possuir residência médica/especialização, morar no município com a não permanência na ESF. No entanto, é clara a associação entre o motivo de entrada na Estratégia e a saída: aqueles profissionais que entraram na ESF motivados pelo salário e oportunidade de emprego foram os que mais deixaram o serviço.

\section{Abstract}

Recruiting, training and establishing a team of professionals are necessary conditions for the consolidation of the Family Health Program (ESF, acronym in Portuguese) as the structuring strategy of Primary Health Care in Brazil. This paper aimed to profile doctors and nurses from the ESF, identifying professionals who settled down to the ESF in the municipality of Praia Grande, São Paulo. All professionals with an university degree were interviewed in 2004-2005 and in 2007 a follow-up was performed with those remained in ESF. All the nurses and $66 \%$ of doctors who had been interviewed previously were still working there after 2-3 years. There was no statistic association between graduation time, medical residency, living in the city and fixedness. However, there is a clear association between the reason for their entry into the ESF and their fixedness: those professionals who got hired from ESF in 2007 aimed a good salary and employment opportunities when were entering in the ESF team.

\section{Resumen}

Dada la unión de la conquista, formación e estancia de profesionales para la consolidación de la Estrategia de Salud Familiar (ESF) como modalidad estructurante de la Atención Primaria de la Salud en Brasil, el presente artículo tiene como objetivo traer el perfil de médicos y enfermeras de la ESF, identificando a los profesionales que se afirmaron a la ESF en el municipio de Praia Grande, São Paulo. Fueron realizadas entrevistas semi-estructuradas con los profesionales de nivel superior en 2004-2005; en 2007 fue verificada la permanencia en la ESF de estos profesionales. No fue observada asociación estadística entre el tiempo de ingresado, poseer residencia médica/ especialización y la renuncia o el despido. Es clara la asociación entre el motivo de la entrada en la Estrategia y la afirmación, aqueles profesionales que entraron en la Estrategia por el sueldo y la oportunidad de empleo fueron los que más dejaron el trabajo.

\footnotetext{
Secretaria de Saúde Pública (SSP) de Praia Grande. Praia Grande (SP), Brasil. ezulianni@gmail.com

${ }^{2}$ Universidade Católica de Santos (UNISANTOS). Santos (SP), Brasil. aylene.bousquat@pesquisador.cnpq.br

${ }^{*}$ Autor correspondente

Conflito de interesses: declararam não haver.

Fonte de financiamento: MS-Decit/CNPq (processo 403238/2004-8)

Recebido em: 08/02/2010

Aprovado em: 05/04/2011
} 


\section{Introdução}

A Estratégia Saúde da Família (ESF) iniciou-se em 1994, para responder ao desafio de ampliar e qualificar a cobertura nesse nível de atenção nos municípios de pequeno porte. Com sua expansão para municípios de médio e grande porte, consolida-se como modelo estruturante da Atenção Primária à Saúde (APS) no Brasil ${ }^{1}$.

Desenvolvida com base nas experiências inovadoras e bem sucedidas de programas isolados, como os dos Estados do Paraná, Mato Grosso do Sul, Ceará e da cidade de Niterói, Rio de Janeiro, a Saúde da Família, entre outras inovações, incorpora o conceito de família como unidade de ação e introduz a noção de adstrição da clientela em base territorial ${ }^{2}$. Sob a perspectiva da organização do trabalho, a ESF assenta-se em um novo modelo fortemente alicerçado no trabalho de equipes multiprofissionais ${ }^{3}$. Essa nova forma de trabalho prevê a existência de profissionais qualificados sob a óptica da atuação generalista; no caso específico de médicos, constitui-se um fator gerador de dificuldades ${ }^{4}$, já que o aparelho formador nacional fundamenta-se, em sua grande maioria, no modelo flexneriano 5 .

Um dos grandes desafios para a implantação da ESF principalmente em municípios de médio e grande porte e nas regiões metropolitanas - reside, portanto, na formação e manutenção das equipes de trabalho, tendo em vista, por um lado, a ausência de profissionais especialmente qualificados para esse tipo de atuação e, por outro, um mercado de trabalho dinâmico, sempre capaz de fornecer alternativas a esses profissionais ${ }^{6}$.

A formação de Recursos Humanos capacitados para uma atuação voltada para a atenção integral à saúde e de práticas que possibilitem as ações de promoção, proteção, prevenção, atenção precoce, cura e reabilitação é um dos principais pontos de constrangimento da ESF. Tais preocupações são facilmente identificadas na leitura dos relatórios das Conferências Nacionais de Recursos Humanos de 1986 e de 1993, assim como na Norma Operacional Básica de Recursos Humanos para o SUS (NOB/RH/SUS, 2002) ${ }^{7}$.

A situação do município de Praia Grande, localizado na Região Metropolitana da Baixada Santista (RMBS), no Estado de São Paulo, não foge à realidade descrita acima. Emancipado politicamente em 1967, sua atividade econômica é voltada para o turismo e serviços, contando com 260.769 habitantes ${ }^{8}$. Em 2006, Viana classificou todos os municípios de São Paulo com mais de 100 mil habitantes segundo seus modelos de APS; Praia Grande foi estratificada no pior cluster, ou seja, municípios que possuem baixa complexidade da rede de atenção à saúde e indicadores sociais menos favoráveis?.
Apesar dessa classificação, a Atenção Primária em Praia Grande vem sendo priorizada desde 1998, quando ocorreu a Implantação da Estratégia de Agentes Comunitários de Saúde (EACS). Em $2001^{10}$, a ESF foi implantada inicialmente com 3 equipes e 15 agentes comunitários de saúde. Esse processo chama a atenção pela sua rapidez decorrente da decisão política de inverter o modelo de atenção à saúde em apenas cinco anos, diferenciando-se, assim, dos demais municípios da RMBS e do Estado de São Paulo.

Em termos de capacidade instalada, essa mudança pode ser dimensionada pelo fato de que, em 2001, o município possuía em sua rede de Atenção Primária 13 Unidades Básicas de Saúde $(\mathrm{UBS})^{10}$. Com a implantação da ESF, as UBSs foram transformadas em Unidades de Saúde da Família, de modo que, em 2006, o município contava com 14 dessas unidades ${ }^{11}$. Dados colhidos junto à Secretaria de Saúde de Praia Grande, em fevereiro de 2008, demonstraram 85\% de cobertura populacional realizada mediante o trabalho de 232 agentes comunitários de saúde e 41 equipes.

No que diz respeito ao regime de trabalho, o município apresentou um diferencial desde o início do processo de implantação, optando por dar estabilidade no emprego aos profissionais da ESF como forma de fomentar a consolidação da Estratégia. Enquanto alguns municípios se utilizavam de diversas vias de contratação (cooperativas de trabalho, Organizações Sociais de Saúde), Praia Grande optou pela realização de processo seletivo e concurso público. Segundo Lopes $^{10}$, "A realização de processo seletivo em 2002 e de concurso público em 2003 para dar cobertura de recursos humanos ao programa demonstra a decisão político administrativa de consolidação do processo de implementação da ESF em Praia Grande". Tanto o processo seletivo quanto o concurso público podem ser identificados como formas de se tentar evitar a precarização do trabalho na ESF.

Dada a centralidade da captação, formação e fixação de profissionais para a consolidação da ESF como modalidade estruturante da APS no País, o presente artigo teve como objetivo traçar o perfil de médicos e enfermeiras da ESF no município de Praia Grande, São Paulo, identificando os profissionais que nela se fixaram.

\section{Metodologia}

Esta investigação, que faz parte do projeto de pesquisa "Avaliação do PSF pela população residente em áreas metropolitanas através de instrumento de coleta de dados informatizado", financiada pelo MS Decit/CNPq (processo 403238/2004-8), foi realizada em dois momentos: em 2004-2005 e em 2007. No primeiro, foram aplicados ques- 
tionários semiestruturados a todos os 76 profissionais de nível superior da ESF naquele momento. Esse questionário foi elaborado a partir dos seguintes eixos: identificação (dados básicos, como: nome, sexo, idade e estado civil), formação profissional (faculdade cursada, instituição pública ou privada, localização da instituição, ano de conclusão do curso, conclusão ou não de residência médica e/ou especialização e em que área), situação funcional (caracterização do vínculo profissional e remuneração) e experiência profissional (capacitação específica e tempo de experiência prévia em Saúde da Família). Uma única questão aberta abordava os motivos de ingresso na ESF e foi codificada tomando-se por referência o ideário da Estratégia e a forma de estruturação do trabalho. Foram definidos três grandes grupos que aglutinavam o conjunto de respostas: identificação com ideário da ESF, identificação com a forma de organização do trabalho (sem plantões e maior autonomia profissional, entre outros) e empregabilidade (incluindo salário).

Em dezembro de 2007, foi efetuada análise de dados documentais na Divisão de Recursos Humanos da Secretaria de Saúde de Praia Grande, a fim de se verificar a permanência na ESF dos mesmos profissionais anteriormente entrevistados. Essa análise foi feita mediante checagem simples das fichas funcionais, sendo colhida a informação sobre o momento do desligamento, caso este tivesse ocorrido.

Deve-se citar o fato de que, na análise documental, efetuada junto à Divisão de Recursos Humanos, não foram incluídos os motivos administrativos que levaram os profissionais a se desligar da ESF. Transferências desses profissionais para outros setores da Secretaria de Saúde ou a ocupação de cargos de chefias dentro da ESF não puderam ser analisadas. No questionário proposto, não foi abordada a influência da realização do introdutório da ESF para os profissionais entrevistados.

A formação profissional, a motivação para ingresso na ESF, o tempo de formado e a realização ou não de residência médica e/ou especialização em Enfermagem foram correlacionados com a permanência na Estratégia Saúde da Família.

Todos os dados obtidos foram digitados em planilhas do programa Microsoft ${ }^{\circledR}$ Excel e, posteriormente, analisados no programa Statistical Package for the Social Sciences (SPSS), versão 15.0. As variáveis qualitativas são apresentadas na forma de frequências simples e as quantitativas, pelas medidas de tendência central e de dispersão. Foram realizadas medidas de associação entre algumas variáveis, sendo que o nível de significância adotado foi $5 \%$, e o teste utilizado foi o Teste Exato de Fisher. Na comparação das médias, utilizou-se o Teste $t$ de Student.

Os preceitos éticos envolvidos nesta investigação foram aprovados pela Comissão de Ética em Pesquisa da Universi- dade Católica de Santos. Os Termos de Consentimento Informado devidamente preenchidos e utilizados na aplicação das entrevistas estão sob a guarda das pesquisadoras.

\section{Resultados}

Observa-se predominância de mulheres entre os profissionais (Gráfico 1), notadamente enfermeiras, e entre os médicos prevalece o sexo masculino.

Em relação à faixa etária, 18 médicos $(44 \%)$ e 20 enfermeiras (57\%) possuem até 35 anos (Tabela 1); a idade média é de 34,74 anos para as enfermeiras e de 36,88 para os médicos. Quanto ao local de moradia, comprovou-se que 16 médicos (39\%) e 15 enfermeiras (43\%) residem na própria cidade.

Quanto ao tempo de formado (Tabela 1), 17 médicos (42\%) tinham 5 ou menos anos de formados. Dos 41 médicos entrevistados, $30(73 \%)$ não possuíam residência médica. Quando a conclusão da residência médica é relacionada ao tempo de formatura, verifica-se que $100 \%$ dos que tinham menos de 5 anos de formados e $50 \%$ dos que possuíam mais de 15 anos de formados não haviam cursado residência médica. Dos 11 profissionais (24\%) que possuem residência médica, $50 \%$ são pediatras, $20 \%$ são cardiologistas, $20 \%$ são cirurgiões gerais e $10 \%$ são obstetras.

No caso das enfermeiras, o percentual com menos de 5 anos de graduação é superior ao dos médicos, chegando a 63\%. Mesmo assim, o quadro de especialização é maior entre elas $(43 \%)$ e a especialização em Saúde Pública é a predominante.

O local da graduação (Tabela 1) é bastante distinto entre médicos e enfermeiras. Enquanto 24 enfermeiras (69\%)

Gráfico 1. Distribuição dos profissionais de nível superior na Estratégia Saúde da Família em Praia Grande, São Paulo, segundo o gênero.

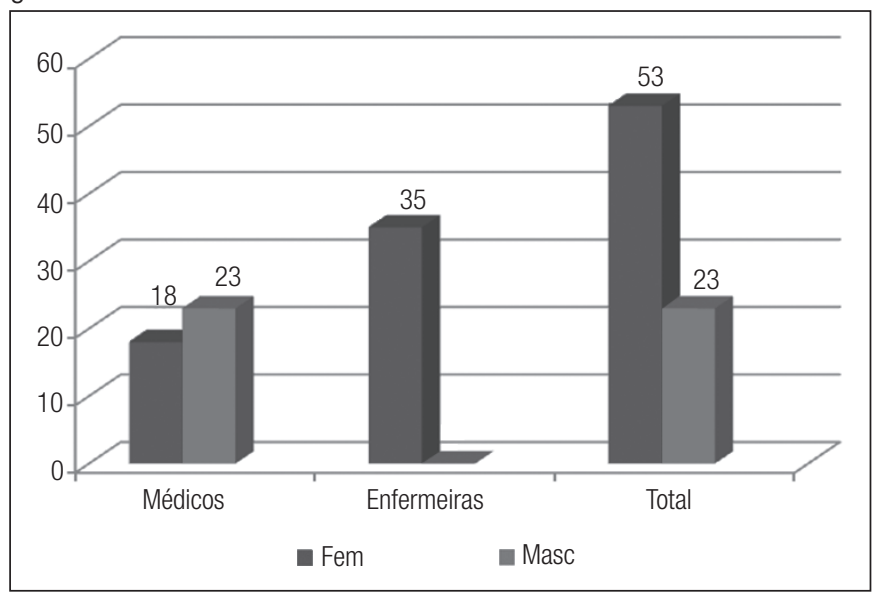


concluíram sua graduação na RMBS, apenas 11 médicos (27\%) formaram-se na região. Vale destacar que $83 \%$ deles e $88 \%$ das enfermeiras são formados por instituições privadas, fato confirmado pela pesquisa do Ministério da Saúde de 2000 para a Região Sudeste ${ }^{12}$. Apenas 6 médicos entrevistados (15\%) referem ter tido noções sobre a ESF durante a graduação.

Doze enfermeiras tiveram noções da ESF durante a graduação. Todos os profissionais da ESF (médicos e enfermeiros) foram admitidos por concurso público, em contraste com a média para a região Sudeste, que é de $12,5 \%{ }^{12}$. A existência de outro vínculo empregatício (Tabela 1) foi referida por $54 \%$ dos médicos e $34 \%$ das enfermeiras.

A maioria dos entrevistados (69\% dos médicos e $66 \%$ das enfermeiras) trabalhava há menos de 2 anos no programa; mesmo assim, as enfermeiras, em grande parte, e quase metade dos médicos tiveram oportunidade de realizar pelo menos 1 curso de reciclagem nos últimos 2 anos. A saúde da mulher e, em especial, o pré-natal são identificados por 21 médicos (44\%) e 7 enfermeiras (20\%) como a área do conhecimento que apresenta maiores dificuldades (Tabela 1).

A opção por trabalhar em Saúde da Família para os médicos baseou-se em duas razões diversas: o salário competitivo no mercado e uma aproximação do ideário da Estratégia como modelo de prática médica. Nesta última, a possibili- dade de participar do pioneirismo da implantação da ESF, contribuindo para a reformulação da atenção primária, apareceu como decisória. Já para as enfermeiras, pesou, fundamentalmente, a aptidão para atuar em Saúde Pública, com ênfase na prevenção e atuação junto à comunidade, além da valorização do trabalho da categoria.

$\mathrm{Na}$ comparação entre médicos e enfermeiras, notou-se diferença estatisticamente significativa na permanência no programa, na realização de cursos de reciclagem e, obviamente, na composição por sexo.

Em relação à rotatividade dos profissionais, em dezembro de 2007, 35 enfermeiras (100\%) e 27 médicos anteriormente entrevistados (66\%) permaneciam atuando na ESF, conforme o Gráfico 2.

Dos 14 médicos (34\%) que deixaram a ESF em Praia Grande no período relatado, 11 (79\%) não possuíam residência e, destes, $6(55 \%)$ tinham menos do que 5 anos de formados. Embora haja um predomínio de profissionais que não cursaram residência médica dentre aqueles que deixaram a ESF, não há associação estatística entre tempo de formado, possuir residência médica, morar no município e a saída da ESF. No entanto, há associação estatística entre o motivo de entrada na ESF e a fixação; aqueles profissionais que entraram no programa pelo salário e oportunidade de emprego foram os que mais deixaram o serviço (Tabela 2).

Tabela 1. Perfil dos médicos e enfermeiros da Estratégia Saúde da Família de Praia Grande, São Paulo - 2004/2005.

\begin{tabular}{|c|c|c|c|c|c|c|}
\hline \multirow[b]{2}{*}{ Característica } & \multicolumn{2}{|c|}{ Médicos } & \multicolumn{2}{|c|}{ Enfermeiras } & \multicolumn{2}{|c|}{ Total } \\
\hline & $\mathrm{n}$ & $\%$ & $\mathrm{n}$ & $\%$ & $\mathrm{n}$ & $\%$ \\
\hline Faixa etária até 35 anos & 18 & 44,00 & 20 & 57,00 & 38 & 50,00 \\
\hline Residem em Praia Grande & 16 & 39,00 & 15 & 43,00 & 31 & 40,79 \\
\hline Não possuem residência médica/especialização enfermagem * & 30 & 73,17 & 20 & 57,14 & 50 & 65,79 \\
\hline Conclusão de curso de graduação há 5 anos ou menos & 17 & 42,00 & 22 & 63,00 & 39 & 51,32 \\
\hline Conclusão de curso de graduação há 15 anos ou mais & 14 & 34,00 & 8 & 23,00 & 22 & 28,95 \\
\hline Formados na RMBS & 11 & 27,00 & 24 & 69,00 & 35 & 46,05 \\
\hline Formados por instituição privada & 34 & 83,00 & 31 & 88,00 & 65 & 85,53 \\
\hline Vínculo empregatício por meio de concurso público & 41 & 100,00 & 35 & 100,00 & 76 & 100,00 \\
\hline Mantêm outro vínculo empregatício & 22 & 54,00 & 12 & 34,00 & 34 & 44,73 \\
\hline Efetuaram reciclagem nos últimos 2 anos* & 19 & 46,34 & 24 & 68,57 & 43 & 56,58 \\
\hline Necessidade de reciclagem em saúde da mulher & 21 & 44,00 & 7 & 20,00 & 28 & 36,48 \\
\hline Formação para a ESF durante a graduação & 6 & 14,63 & 12 & 34,28 & 18 & 23,37 \\
\hline Permaneciam na ESF (dezembro de 2007) * & 27 & 65,90 & 35 & 100,00 & 62 & 81,58 \\
\hline
\end{tabular}

RMBS: Região Metropolitana da Baixada Santista; ESF: Estratégia Saúde da Família. ${ }^{\star} p<0,05$. 
Gráfico 2. Evolução do número de profissionais médicos e enfermeiras na Estratégia Saúde da Família em Praia Grande, São Paulo (2004-2005 a 2007).

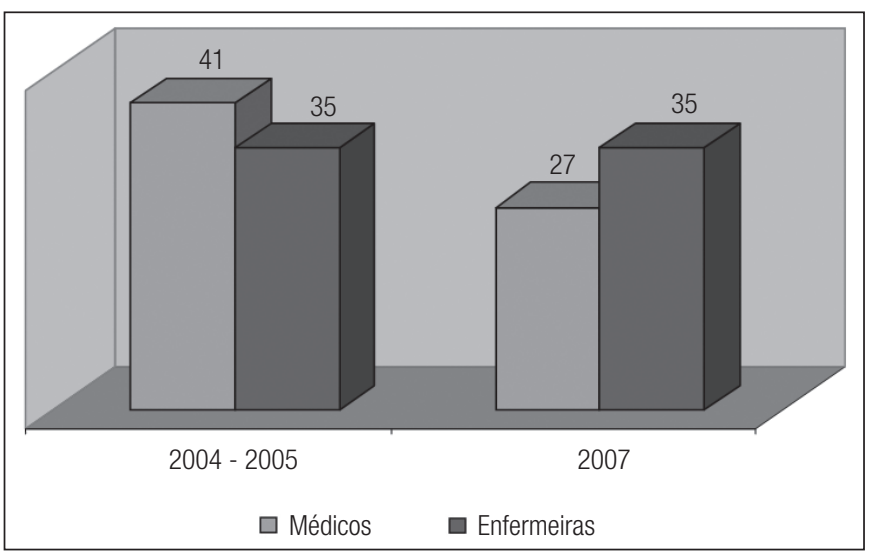

\section{Discussão}

Ao serem comparadas as características dos médicos entrevistados com o perfil traçado para os médicos da ESF pelo Ministério da Saúde (2000), constata-se predomínio de representantes do sexo masculino. Em Praia Grande, chama a atenção a presença de maior percentual de médicos sem especialização (73 contra $54 \%$ da média da Região Sudeste) e com outro vínculo empregatício (54 contra 44\%). A distribuição por especialidades, dos poucos que a possuem, segue perfil semelhante do padrão nacional dos médicos do PSF em $2000^{13}$. Estes dados sugerem a baixa qualificação formal dos médicos da ESF no município em questão. No caso das enfermeiras, o perfil local é muito semelhante ao nacional quanto à distribuição por sexo, existência de outro vínculo empregatício e especialização.

No período citado, verificou-se exatamente a fase inicial de implantação da ESF em Praia Grande; a equipe de médicos e enfermeiros era composta por profissionais jovens e, geralmente, sem especialização nem conhecimentos prévios sobre a filosofia de trabalho proposta. A constatação de que, durante a graduação, apenas $15 \%$ dos médicos tiveram informações sobre a ESF leva-nos a citar Campos e Belisário ${ }^{14}$, que destacam que um dos principais óbices à consecução dos relevantes objetivos de se reestruturar o modelo assistencial é a formação médica. Esses autores concluem que os profissionais atuais " $[\ldots .$.$] são, portanto, incapazes de transcender o$ espaço do consultório médico e propor diagnóstico e intervenções sobre o coletivo, numa abordagem integralizadora, sem a qual dificilmente se lograrão avanços significativos". Marsiglia ${ }^{15}$ também ressalva que o espaço da comunidade tem lógicas muito distintas daquelas que normalizam a organização dos hospitais de ensino e que as atribuições das equipes multiprofissionais são amplas e complexas, exigin-
Tabela 2. Associação entre a motivação de ingresso e fixação dos profissionais da Estratégia Saúde da Família de Praia Grande, São Paulo após 03 anos - 2007.

\begin{tabular}{lrrrr} 
& \multicolumn{4}{c}{ Fixação } \\
\cline { 2 - 5 } Motivação & \multicolumn{1}{c}{ Sim } & Não \\
& $\mathrm{n}$ & \multicolumn{1}{c}{$\%$} & $\mathrm{n}$ & $\%$ \\
\hline Identificação com os ideais do programa * & 42 & 85,5 & 7 & 14,5 \\
Identificação com a forma de organização do trabalho * & 13 & 100,0 & 0 & 0,0 \\
Salário e oportunidade de emprego & 7 & 50,0 & 7 & 50,0 \\
\hline${ }^{*} \mathrm{P}<0,05$ & & &
\end{tabular}

do dos profissionais de saúde novos e continuados conhecimentos e autonomia, entendida como capacidade de análise dos problemas.

Ao reconhecer esse quadro e na tentativa de promover a homogeneização, ainda que básica, dos conhecimentos sobre a ESF, o Ministério da Saúde propõe a realização do Curso Introdutório para Estratégia Saúde da Família ${ }^{16}$ para todas as equipes contratadas. Entretanto, questionase se esse tipo de complementação no processo de formação é suficiente para garantir a adesão profissional a uma nova forma de trabalho e para assegurar que este seja capazes de se aproximar do profissional desejado descrito no parágrafo anterior.

Além disso, chama a atenção o fato de que, dentre o grupo dos entrevistados, um percentual maior de enfermeiras (34\%) do que de médicos (15\%) teve noções sobre a ESF durante a graduação. Ainda que os percentuais sejam baixos para ambos, demonstrando falta de sintonia ou de priorização da ESF nas escolas da área da saúde, o quadro é um pouco menos acentuado nas escolas de Enfermagem. Aparentemente, o peso da expansão da ESF no mercado de trabalho para as enfermeiras é maior do que para os médicos, importância esta que reflete muito rapidamente no aparelho formador. Ao se levar em conta que 34 médicos (83\%) e 31 enfermeiras (88\%) da ESF de Praia Grande cursaram sua graduação em cursos mantidos por instituições privadas, caberia indagar qual o papel do aparelho formador público diante dessa nova Estratégia de reorganização do Sistema de Saúde.

A Política Nacional de Educação Permanente, por intermédio dos diversos Polos de Educação, promoveu diversos cursos de especialização em Saúde da Família no Estado de São Paulo e no Brasil, com o objetivo de qualificar a Atenção Básica. Na RMBS, foram realizadas, no período citado, capacitações sobre os temas Saúde da Mulher e Saúde Mental para as equipes de Saúde da Família, mas que atingiram baixo percentual de participantes da ESF local ${ }^{17}$.

Quando questionados sobre a necessidade de aprimoramento nas áreas da Saúde da Mulher, da Criança e do Adulto, a Saúde da Mulher desponta como a principal para 
$44 \%$ dos médicos e $20 \%$ das enfermeiras. Cabe registrar que Praia Grande tem elevado coeficiente de mortalidade infantil, tomando por comparação a média estadual ${ }^{18}$. As dificuldades na implementação da saúde materno-infantil também foram relatas em estudo conduzido por Silva et al. ${ }^{19}$ no município de Duque de Caxias, no Rio de Janeiro, que definiu a ESF como estruturadora da APS e contratou suas equipes via concursos públicos.

A qualificação inadequada dos profissionais pode gerar inúmeras consequências. Entre estas, podemos salientar a baixa qualidade da assistência prestada, dificultando a melhoria das condições de saúde da população. Ademais, a inexistência de programas de capacitação que permitam o crescimento e aprimoramento profissional pode contribuir para a evasão desses profissionais, que vislumbrariam a necessidade de complementar sua formação acadêmica. Ademais, o dinamismo de oportunidades no mercado de trabalho para os médicos nas regiões economicamente mais ativas, conforme descrito por Negri et al ${ }^{20}$, pode contribuir para a demissão voluntária dos médicos, que facilmente se realocam no mercado.

Uma das limitações deste estudo foi não se determinar com exatidão as causas que levaram ao afastamento desses profissionais. Entretanto, esse fenômeno não é exclusivo do município em questão. Estudo realizado no Canadá por Geneau et al. ${ }^{21}$ demonstra a preocupação dos promotores de políticas públicas no que diz respeito à rotatividade dos "GPs" (os médicos gerais que atuam como porta de entrada no sistema canadense). As possíveis causas citadas por esses autores para o Canadá (a satisfação no trabalho, como aumento da carga horária, pressões diárias e a retirada da autonomia profissional) são questões centrais na prática médica e mereciam ser abordadas em outros estudos no nosso país.

Em relação à equipe de enfermeiras, o quadro relativo à fixação das profissionais foi totalmente diverso: $100 \%$ das que foram entrevistadas em 2005 permaneciam atuantes na ESF de Praia Grande em dezembro de 2007, denotando expressiva adesão à mesma, possivelmente impulsionadas pela melhor discussão metodológica do novo modelo durante a graduação, pela estabilidade garantida pelo concurso público e pela valorização do trabalho do enfermeiro. Nascimento e Nascimento ${ }^{22}$ enfatizam o aspecto da valorização do trabalho do enfermeiro por meio "[...] do reconhecimento pela comunidade, da maior autonomia em sua área de atuação, da oportunidade em que além de utilizarem o saber clínico na prática assistencial, utilizam o saber epidemiológico em situações de risco, assim como também o enfoque educativo nas ações de promoção e prevenção à saúde".

\section{Conclusões}

O expressivo número de profissionais sem residência ou especialização sugere perfil de profissionais com baixa qualificação, alertando aos gestores a necessidade de investimentos ainda maiores na capacitação daqueles. Esse cenário denota importantes constrangimentos para a implementação e o desenvolvimento da ESF.

A contratação de profissionais sem a formação completa também atua como constrangimento para a continuidade do programa, visto que muitos deles, durante as entrevistas realizadas em 2004-2005, informaram que pretendiam se aprimorar.

Todavia, é evidente que a maior dificuldade na fixação ocorre entre os médicos. Nem mesmo a estabilidade garantida por concurso público, um dos principais diferenciais de Praia Grande, foi suficientemente capaz de garantir a permanência deles no município. Isso pode ser explicado pela dinâmica do mercado de trabalho médico, que permite inúmeras possibilidades de inserção profissional, possibilitando não só a compatibilidade com outro vínculo empregatício, mas também a agilidade na troca do emprego. Os dados encontrados sugerem que é a afinidade ao ideário da ESF que prende o médico nesse local de trabalho.

Outro aspecto que merece destaque se refere ao local de moradia dos profissionais, pois a maior parte deles não mora na cidade em que trabalha. Tal fato caracteriza, por um lado, a lógica metropolitana de fluxos de profissionais na região e, por outro, o que Castells ${ }^{23}$ indica como a "necessidade" daqueles portadores de alta qualificação morarem em áreas com maior disponibilidade de recursos culturais, econômicos e sociais.

A consolidação e a ampliação da ESF como eixo estruturante da APS no Brasil, sem dúvida, têm como um de seus maiores desafios fixar profissionais nas distintas oportunidades do mercado de trabalho. Isso implica na elaboração e implementação de políticas específicas e ousadas para esse segmento, que consigam que os profissionais de Saúde da Família sejam vistos com valorização e reconhecimento por seus pares e pela sociedade e que suas expectativas de renda e formação possam ser contempladas.

\section{Referências}

1. Souza HM. Saúde da família: desafios e conquistas. In: Negri B, Viana AL. O sistema único de saúde em dez anos de desafios. São Paulo: Centro de Estudos Augusto Leopoldo Ayrosa Galvão; 2002. p. 221-40

2. Viana ALD, Dal Poz MR. A reforma do sistema de saúde no Brasil e o Programa de Saúde da Família. Physis. 1998; 8(2): 11-48.

3. Brasil. Ministério Da Saúde. I Conferência Nacional de Recursos Humanos em Saúde. Relatório Final. Brasília: Secretaria de Recursos Humanos, Ministério da Saúde; 1986. 
4. Brasil. Ministério Da Saúde. II Conferência Nacional de Recursos Humanos em Saúde. Relatório Final. Brasília: Coordenação Geral de Recursos Humanos para o SUS. Ministério da Saúde; 1993.

5. Brasil. Ministério Da Saúde. Princípios e diretrizes para a NOB/RH-SUS. Brasília: Conselho Nacional de Saúde; 2002.

6. Bousquat A, Cohn A, Elias PE. O PSF e a dinâmica urbana das grandes cidades. In: Viana ALD, Elias PPE, Ibañez N. (orgs.). Proteção social. Dilemas e desafios. São Paulo: Hucitec; 2005. p. 244-65.

7. Brasil, Ministério Da Saúde, Conselho Nacional de Saúde (CNS) Princípios e Diretrizes para a Norma Operacional Básica de Recursos Humanos para o SUS (NOB/RH-SUS). $2^{a}$. ed. rev. atual. Brasilia: Ministério da Saúde; 2003. Disponível em: http://bvsms.saude.gov.br/ bvs/publicacoes/principios_diretrizes_NOB_2003.pdf

8. IBGE. Censo Demográfico de 2010. [acesso em 2010 Mar 20]. Disponível em: http://www.ibge.gov.br/cidadesat/topwindow.htm?1

9. São Paulo (Estado). Programa de Expansão e Consolidação da Saúde da Família. Caracterização dos municípios paulistas com população superior a 100 mil habitantes. São Paulo: Centro de Estudos de Cultura Contemporânea. Consórcio Medicina USP, 2006.

10. Lopes EZ. A implantação do programa de saúde da família no município de Praia Grande, Região Metropolitana da Baixada Santista [dissertação]. Santos: Universidade Católica de Santos; 2005.

11. Secretaria de Saúde de Praia Grande. Relatório Anual de Gestão. Praia Grande: Prefeitura Municipal de Praia Grande; 2006.

12. Machado MH. Os médicos no Brasil: um retrato da realidade. Rio de Janeiro: Fiocruz; 1997.

13. Brasil. Ministério da Saúde. Secretaria de Políticas de Saúde. Perfil dos médicos e enfermeiros do programa de saúde da família no Brasil. Relatório final: Região Sudeste. Brasília: Ministério da Saúde; 2000.
14. Campos FE, Belisário SA. O programa de saúde da família e os desafios para a formação profissional e a educação continuada. Interface Comunic, Saúde, Educ. 2001; 9: 133-42.

15. Marsiglia RMG. Instituições de ensino e o Programa Saúde da Família: o que mudou? Rev Bras Saúde Família. 2004; 5(7): 30-41.

16. Brasil. Ministério Da Saúde. Portaria GM 2527, de 19 de outubro de 2006. Brasília: Ministério da Saúde; 2006.

17. Direção Regional de Saúde DIR XIX, Pólo de Educação Permanente para o SUS da Baixada Santista, Relatório Anual de Atividades; 2005.

18. Bousquat A, Pereira LA, Braga A, Lorandi PA, Vieira MRS, Elias PEM, et al. Caracterização da mortalidade neonatal e perinatal na Região Metropolitana da Baixada Santista. Relatório de Pesquisa; Santos: CNPq; 2007. Processo 403593/2004-2.

19. Silva, ACMA, Villar MAM, Wuillaume SM, Cardoso MHCA. Perspectivas de médicos do Programa Saúde da Família acerca das linhas de cuidado propostas pela Agenda de Compromissos para a Saúde Integral da Criança e Redução da Mortalidade Infantil. Cad Saúde Pública. 2009; 25(2) : 349-58.

20. Negri B, Faria R, Viana ALD. Recursos Humanos em Saúde. Política, Desenvolvimento e Mercado de Trabalho. Campinas: Unicamp; 2002.

21. Geneau R, Lehoux P, Pineault R, Lamarche PA. Primary care practice a la carte among GPs: using organizational diversity to increase job satisfaction. Fam Pract. 2007; 24(2): 138-44.

22. Nascimento MS, Nascimento MAA. Prática da enfermeira no Programa de Saúde da Família: a interface da vigilância da saúde versus as ações programáticas em saúde. Ciênc. saúde coletiva. 2005; 10(2): 333-45.

23. Castells M. A sociedade em rede. v. 1. São Paulo: Paz e Terra; 1999. 